\title{
THE GLUTARALDEHYDE COAGULATION TEST (GCT) USED TO PREDICT HEALTH STATUS IN DAIRY HERDS
}

\author{
Trine Tøllbøll ${ }^{1}$, and Rolf Jess Jørgensen ${ }^{2}$ \\ ${ }^{1}$ Royal Veterinary and Agricultural University, Department of Clinical Studies, Large Animal Surgery, \\ Dyrlægevej 48, DK-1870 Frb. C, Denmark; ${ }^{2}$ Royal Veterinary and Agricultural University, Department of \\ Clinical Studies, Large Animal Medicine, Dyrlægevej 88, DK-1870 Frb.C, Denmark.
}

As dairy farming intensifies the unit of interest is changing from the individual cow to the herd. It has become increasingly important to identify problem herds and a great challenge met by veterinary practitioners is the demand to estimate health status in herds in a way that is directly comparable between herds as well as between veterinarians. There is therefore a great need for methods that provide a consistent and objective measure of health status at herd level.

The GCT test is used to estimate the level of immunoglobulins and fibrinogen semiquantitatively in bovine whole blood and thereby it detects inflammatory conditions in individual animals.

The objective of this study was to apply the glutaraldehyde coagulation test (GCT) at the herd level as a health indicator to assess the level of inflammatory disease in Danish dairy herds.

A total of 420 animals originating from 10 different dairy herds - each contributing 37 to 51 cows were included in the study. All cows were GCT tested and subjected to a standardized superficial clinical examination of udder, limbs and skin lesions and scored accordingly. The clinical examination was designed to detect diseases considered of major importance to animal welfare.

Herd indexes for the GCT results and the clinical scores were calculated and were found to correlate well.

To test the performance of the GCT in individual animals the odds of a positive GCT were calculated and found to increase significantly with increasing clinical score.

The data indicate that testing approximately 40 cows gives a good indication of the level of inflammatory conditions present within a herd. These results support the possible application of the GCT as a tool in future herd health and welfare evaluations. As such it can be used to identify problem herds or groups within a herd where further intervention is needed.

It must be stressed that a test like the GCT never should stand on its own and should be used to support - not replace - a trained practitioner in the surveillance of herd health and welfare.

The conclusions drawn above are based on the finding that the GCT is a suitable predictor of superficial inflammatory conditions in individual cows. 